Stud. Univ. Babeş-Bolyai Math. 64(2019), No. 3, 305-312

DOI: http://dx.doi.org/10.24193/subbmath.2019.3.02

\title{
On two modified Phillips operators
}

\author{
Gancho Tachev
}

Dedicated to Professor Heiner Gonska on the occasion of his 70th anniversary.

\begin{abstract}
In this note we introduce two new modified Phillips operators $G_{n}^{1}$ and $G_{n}^{2}$. We obtain direct estimates for approximation of bounded continuous functions, defined on $[0, \infty)$ by $G_{n}^{1}$, as well as for approximation of unbounded continuous functions by $G_{n}^{2}$. We improve some previous results on this topic.
\end{abstract}

Mathematics Subject Classification (2010): 41A25, 41A36.

Keywords: Phillips operators, exponential functions, quantitative results.

\section{Introduction}

The Phillips operator [20] is defined as

$$
S_{n}(f ; x)=n \sum_{k=1}^{\infty} e^{-n x} \frac{(n x)^{k}}{k !} \int_{0}^{\infty} e^{-n t} \frac{(n t)^{k-1}}{(k-1) !} f(t) d t+e^{-n x} f(0) .
$$

These operators preserve constant as well linear functions. Some approximation results on these operators- commutativity, direct and strong converse inequalities, inverse estimates, linear combinations etc. have been discussed in $[8,21,22,11,14,23,24$, $12,15,13]$. Usually we denote the basis functions by

$$
s_{n, k}(x)=e^{-n x} \frac{(n x)^{k}}{k !} .
$$

In the last decade a lot of papers appeared devoted to such modifications of classical positive linear operators, which preserve certain exponential type functions. For the new modified Szász-Mirakyan operators we refer the reader to recent papers $[1,2,3,5$, 4].In the same way, Gupta and Tachev [12] considered Phillips type operators fixing $e^{-t}$ and $e^{A t}, A \in \mathbb{R}$, but not both together. Very recently Gupta and Lopez-Moreno in [13] defined a modification of the Phillips so as to fix both $e^{a t}$ and $e^{b t}$ for any two real numbers, different or not , $a, b \in \mathbb{R}$ and studied their approximation behaviour. In most of the results, mentioned above - the modification consists of modelling the 
basis functions (for example $s_{n, k}(x)$ in case of Szász-Mirakyan operator) such that certain exponential functions are reproduced. The second approach is to modify the argument of the function, to be approximated. The start of this method was given by the work of King [17] on the classical Bernstein operator and further developed for the Szász-Mirakyan operator in [7]. To combine the both methods Aral, Inoan and Raşa generalized Szász-Mirakyan operator in [3] introducing the function $\rho(x)$ in both places - in basis functions, as well as in the argument of the approximated function. Our method is different from all, mentioned above and we modify simultaneously the basis functions $s_{n, k}(x)$ and multiply the approximated function $f$ by $e^{A t}, A=-1,1$. The case of arbitrary $A \in \mathbb{R}$ is similar and we omit the details. In [12] the following modification of Phillips operator was introduced

$$
P_{n}(f ; x)=n \sum_{k=1}^{\infty} e^{-n \alpha_{n}(x)} \frac{\left(n \alpha_{n}(x)\right)^{k}}{k !} \int_{0}^{\infty} e^{-n t} \frac{(n t)^{k-1}}{(k-1) !} f(t) d t+e^{-n \alpha_{n}(x)} f(0) .
$$

By Lemma 1 in [12] it follows for $A \in \mathbb{R}$

$$
P_{n}\left(e^{A t} ; x\right)=e^{\frac{A n \alpha_{n}(x)}{n-A}} .
$$

It was shown in [12] that if we choose

$$
\alpha_{n}(x)=\frac{x(n+1)}{n}, x \in[0, \infty),
$$

then

$$
P_{n}\left(e^{-t} ; x\right)=e^{-x} .
$$

If we choose

$$
\alpha_{n}(x)=x\left(1-\frac{A}{n}\right), A>0
$$

then from [12] we have

$$
P_{n}\left(e^{t} ; x\right)=e^{x} .
$$

Further we restrict ourselves in (1.5) for $A=1$. Now we define our two modified Phillips operators as follows:

$$
G_{n}^{1}(f ; x)=e^{-x}\left(n \sum_{k=1}^{\infty} s_{n, k}\left(\alpha_{n}(x)\right) \int_{0}^{\infty} s_{n, k-1}(t) f(t) e^{t} d t+e^{-n \alpha_{n}(x)} f(0)\right),
$$

where $\alpha_{n}(x)=x\left(1-\frac{1}{n}\right)$. The second modification is given by

$$
G_{n}^{2}(f ; x)=e^{x}\left(n \sum_{k=1}^{\infty} s_{n, k}\left(\alpha_{n}(x)\right) \int_{0}^{\infty} s_{n, k-1}(t) f(t) e^{-t} d t+e^{-n \alpha_{n}(x)} f(0)\right),
$$

where

$$
\alpha_{n}(x)=x\left(1+\frac{1}{n}\right) .
$$

Further we adopt that 2 in definition of $G_{n}^{2}$ serves as an index and not as a power factor. It is clear that

$$
G_{n}^{1}(f ; x)=e^{-x} P_{n}\left(f(t) e^{t}, x\right),
$$


with $\alpha_{n}(x)$ from (1.7) and

$$
G_{n}^{2}(f ; x)=e^{x} P_{n}\left(f(t) e^{-t}, x\right),
$$

with $\alpha_{n}(x)$ from (1.8). We apply the operator $G_{n}^{1}$ to approximate bounded continuous function $f \in C^{\star}[0, \infty)$-the subspace of real-valued continuous functions, which possess finite limit at infinity, endowed with the uniform norm. The operator $G_{n}^{2}$ will be used to approximate unbounded continuous $f \in C[0, \infty)$. In Section 2 we study the approximation order of $G_{n}^{1}$ and compare our direct estimate with previous known results on this topic. In Section 3 using some ideas from [2] we discuss an uniform error estimate for the operator $G_{n}^{2}$ measured in weighted norm.

\section{Estimate for $G_{n}^{1}$.}

In Boyanov, Veselinov -[6] the uniform convergence of linear positive operators was established. Later Holhos in [16] established the following quantitative estimate for a sequence of linear positive operators:

Theorem A. [16] If a sequence of linear positive operators $L_{n}: C^{*}[0, \infty) \rightarrow C^{*}[0, \infty)$ satisfy the equalities

$$
\begin{aligned}
& \left\|L_{n} e_{0}-1\right\|_{[0, \infty)}=\alpha_{n} \\
& \left\|L_{n}\left(e^{-t}\right)-e^{-x}\right\|_{[0, \infty)}=\beta_{n} \\
& \left\|L_{n}\left(e^{-2 t}\right)-e^{-2 x}\right\|_{[0, \infty)}=\gamma_{n}
\end{aligned}
$$

then

$$
\left\|L_{n} f-f\right\|_{[0, \infty)} \leq\|f\|_{\infty} \cdot \alpha_{n}+\left(2+\alpha_{n}\right) \cdot \omega^{*}\left(f, \sqrt{\alpha_{n}+2 \beta_{n}+\gamma_{n}}\right), f \in C^{*}[0, \infty) .
$$

The modulus of continuity used in the above theorem is defined as:

$$
\omega^{*}(f, \delta):=\sup _{\substack{e^{-x}-e^{-t} \mid \leq \delta \\ x, t \geq 0}}|f(t)-f(x)| .
$$

Our first result states the following

Theorem 2.1. For $f \in C^{*}[0, \infty)$ we have

$$
\left\|G_{n}^{1} f-f\right\|_{C[0, \infty)} \leq 2 \omega^{*}\left(f, \sqrt{\gamma_{n}}\right)
$$

where

$$
\gamma_{n}=\left\|G_{n}^{1}\left(e^{-2 t} ; x\right)-e^{-2 x}\right\|_{C[0, \infty)}=\frac{1}{n+1}\left(\frac{n+1}{n}\right)^{-n}<\frac{1}{2(n+1)} .
$$

Proof. From definition of the operator $G_{n}^{1}-(1.9)$ and (1.4) it follows that

$$
G_{n}^{1}(1 ; x)=1, G_{n}^{1}\left(e^{-t} ; x\right)=e^{-x},
$$

i.e. $\alpha_{n}=\beta_{n}=0$. Simple calculations and (1.3) imply (2.2). Now the proof follows immediately from Theorem A. 
Our further uniform estimate is based on the suitable transformation which reduces the uniform approximation problem on $C^{*}[0, \infty)$ to that one on $C[0,1]$. This observation was developed by Gonska in [10] and by Paltanea in [18] and quantitative results were obtained in [19]. In [1] it was shown that the spaces $\left(C^{*}[0, \infty),\|\cdot\|_{C[0, \infty)}\right)$ and $\left(C[0,1],\|\cdot\|_{C[0,1]}\right)$ are isometrically isomorphic. If we define

$$
\psi(t)=e^{-t}, t \in[0, \infty)
$$

and $S^{*}: C^{*}[0, \infty) \rightarrow C^{*}[0, \infty)$ is a positive linear operator, reproducing the constant functions, then the following statement was proved in [1] (see Theorem 9 there):

Theorem B. [1] If $S^{*}: C^{*}[0, \infty) \rightarrow C^{*}[0, \infty)$, then for all $f^{*} \in C^{*}[0, \infty)$ and $0<h \leq$ $\frac{1}{2}$, the following inequality holds

$$
\begin{gathered}
\left\|S^{*} f^{*}-f^{*}\right\|_{C[0, \infty)} \leq \frac{1}{h}\left\|S^{*}(\psi)-\psi\right\|_{C[0, \infty)} \omega_{1}(f, h) \\
+\left[1+\frac{1}{2 h^{2}}\left(\left\|S^{*}\left(\psi^{2}\right)-\psi^{2}\right\|_{C[0, \infty)}+2\left\|S^{*}(\psi)-\psi\right\|_{C[0, \infty)}\right)\right] \omega_{2}(f, h) .
\end{gathered}
$$

Here $f=f^{*} \circ \psi^{-1}$, i.e. $f \in C[0,1]$ and $\omega_{1}, \omega_{2}$ are the usual first and second order moduli of continuity. Our second result states the following:

Theorem 2.2. For $f^{*} \in C^{*}[0, \infty)$ we have

$$
\left\|G_{n}^{1} f^{*}-f^{*}\right\|_{C[0, \infty)} \leq \frac{5}{4} \omega_{2}\left(f, \frac{1}{\sqrt{n+1}}\right),
$$

where $f=f^{*} \circ \psi^{-1}$.

Proof. The proof follows directly from Theorem B, (2.2) and the fact that $G_{n}^{1}$ reproduces constant and $e^{-t}=\psi(t)$.

\section{Estimates for $G_{n}^{2}$}

In this section we study the approximation of unbounded functions, satisfying certain exponential growth by the operator $G_{n}^{2}$ with

$$
\alpha_{n}(x)=x\left(1+\frac{1}{n}\right) .
$$

Set $\varphi(x)=1+e^{x}, x \in \mathbb{R}^{+}$and consider the following weighted spaces

$$
\begin{gathered}
B_{\varphi}\left(\mathbb{R}^{+}\right)=\left\{f: \mathbb{R}^{+} \rightarrow \mathbb{R}:|f(x)| \leq M_{f} \cdot \varphi(x), x \geq 0\right\}, \\
C_{\varphi}\left(\mathbb{R}^{+}\right)=C\left(\mathbb{R}^{+}\right) \cap B_{\varphi}\left(\mathbb{R}^{+}\right), \\
C_{\varphi}^{k}\left(\mathbb{R}^{+}\right)=\left\{f \in C_{\varphi}\left(\mathbb{R}^{+}\right): \lim _{x \rightarrow \infty} \frac{f(x)}{\varphi(x)}=k_{f} \text { exists and it is finite, }\right\}
\end{gathered}
$$

where $M_{f}, k_{f}$ are constants depending on $f$. All three spaces are normed with the norm

$$
\|f\|_{\varphi}=\sup \frac{|f(x)|}{\varphi(x)} .
$$


Obviously we have

$$
G_{n}^{2}(1 ; x)=1, \text { and } G_{n}^{2}\left(e^{t} ; x\right)=e^{x}
$$

Further from (1.10) we obtain

$$
\begin{aligned}
G_{n}^{2}(f ; x) & =e^{x} P_{n}\left(\frac{f(t)}{1+e^{t}} \cdot\left(1+e^{t}\right) e^{-t} ; x\right) \\
& \leq e^{x}\|f\|_{\varphi} P_{n}\left(e^{-t}+1 ; \alpha_{n}(x)\right) \\
& =e^{x}\|f\|_{\varphi}\left(e^{-x}+1\right) \\
& =\|f\|_{\varphi}\left(1+e^{x}\right) .
\end{aligned}
$$

Consequently we have

$$
\left\|G_{n}^{2} f\right\|_{\varphi} \leq\|f\|_{\varphi}
$$

and we conclude that $G_{n}^{2}$ maps $C_{\varphi}\left(\mathbb{R}^{+}\right)$to $C_{\varphi}\left(\mathbb{R}^{+}\right)$. Following the general result obtained by Gadziev -[9] if we choose as a weight function $\varphi(x)=1+e^{2 a x}, a=1$, instead of $\varphi(x)=1+e^{x}$, to conclude that for each function $f \in C_{\varphi}^{k}\left(\mathbb{R}^{+}\right)$

$$
\lim _{n \rightarrow \infty}\left\|G_{n}^{2} f-f\right\|_{\varphi}=0
$$

it is enough to verify the three conditions

$$
\lim _{n \rightarrow \infty}\left\|G_{n}^{2} e^{i t}-e^{i x}\right\|_{\varphi}=0, i=0,1,2 .
$$

For $i=0,1$ this follows from (3.1). Unfortunately the condition for $i=2$ is not satisfied. Indeed we have

$$
\begin{aligned}
G_{n}^{2}\left(e^{2 t} ; x\right) & =e^{x} P_{n}\left(e^{2 t} e^{-t} ; x\right)=e^{x} P_{n}\left(e^{t} ; \alpha_{n}(x)\right) \\
& =e^{x} \cdot e^{\frac{n}{n-1} x \frac{(n+1}{n}}=e^{x \frac{2 n}{n-1}} .
\end{aligned}
$$

Therefore

$$
\frac{G_{n}^{2}\left(e^{2 t} ; x\right)-e^{2 x}}{1+e^{2 x}}=\frac{e^{2 x \frac{n}{n-1}}-e^{2 x}}{1+e^{2 x}} \rightarrow \infty,
$$

when $x \rightarrow \infty$ for $n \geq 2$. This is the reason to choose $\varphi(x)=1+e^{x}$ instead of $\varphi(x)=1+e^{2 x}$. Now, according to the Korovkin- type theorem, established in [9] we need to verify

with

$$
\lim _{n \rightarrow \infty}\left\|G_{n}^{2}\left(e^{\frac{t}{2}} ; x\right)-e^{\frac{x}{2}}\right\|_{\varphi}=0
$$

and using (1.3) we calculate

$$
\alpha_{n}(x)=\frac{x(n+1)}{n}
$$

$$
\begin{aligned}
G_{n}^{2}\left(e^{\frac{t}{2}} ; x\right) & =e^{x} P_{n}\left(e^{-\frac{t}{2}} ; \alpha_{n}(x)\right)=e^{x} \cdot e^{-\frac{1}{2} \frac{n}{\left(n+\frac{1}{2}\right)} \frac{n+1}{n} x} \\
& =e^{x \cdot \frac{n}{2 n+1}}
\end{aligned}
$$

Therefore

$$
\frac{G_{n}^{2}\left(e^{\frac{t}{2}} ; x\right)-e^{\frac{x}{2}}}{1+e^{x}}=\frac{e^{x \cdot \frac{n}{2 n+1}}-e^{\frac{x}{2}}}{1+e^{x}}=\frac{y^{\frac{2 n}{2 n+1}}-y}{1+y^{2}}:=g(y),
$$

where we set $e^{\frac{x}{2}}=y \in[1, \infty)$. 
Hence

$$
g(y)=\frac{y^{2}}{\left(1+y^{2}\right)}\left[y^{-\frac{2 n+2}{2 n+1}}-y^{-1}\right]
$$

Consequently

$$
\begin{aligned}
\|g\|_{C[1, \infty)} & \leq\left\|\frac{y^{2}}{1+y^{2}}\right\|_{C[1, \infty)}\left\|y^{-\frac{2 n+2}{2 n+1}}-y^{-1}\right\|_{C[1, \infty)} \\
& \leq\left\|y^{-\frac{2 n+2}{2 n+1}}-y^{-1}\right\|_{C[1, \infty)}
\end{aligned}
$$

Simple computations imply

$$
\begin{aligned}
\left\|y^{-\frac{2 n+2}{2 n+1}}-y^{-1}\right\|_{C[1, \infty)} & =\frac{1}{(2 n+1)}\left(1-\frac{1}{2 n+2}\right)^{2 n+2} \\
& <\frac{1}{2(2 n+1)}
\end{aligned}
$$

where we used

$$
\lim _{n \rightarrow \infty}\left(1-\frac{1}{2 n+2}\right)^{2 n+2}=e^{-1}<\frac{1}{2} .
$$

It is clear that (3.2) follows from (3.3), (3.4), (3.5). Our next statement is:

Theorem 3.1. For each function $f \in C_{\varphi}^{k}\left(\mathbb{R}^{+}\right), \varphi(x)=1+e^{x}$ we have

$$
\lim _{n \rightarrow \infty}\left\|G_{n}^{2} f-f\right\|_{\varphi}=0
$$

Proof. The proof is straightforward corollary from (3.1) and (3.2).

Remark 3.2. If in the definition of $G_{n}^{2}$ from (1.10) instead of

$$
\alpha_{n}(x)=x\left(1+\frac{1}{n}\right)
$$

we use

$$
\alpha_{n}(x)=x\left(1-\frac{1}{n}\right)
$$

then (1.3) implies in this case

$$
G_{n}^{2}\left(e^{t} ; x\right)=e^{x}, G_{n}^{2}\left(e^{2 t} ; x\right)=e^{2 x} .
$$

But for the third test function of Korovkin-type theorem $e^{0 t}=1$ simple calculations show

$$
\lim _{n \rightarrow \infty}\left\|G_{n}^{2}(1 ; x)-1\right\|_{\varphi}=\infty,
$$

with $\varphi(x)=1+e^{2 x}$. Similar examples for nonconvergence in weighted norm can be found for Phillips operator in [13].

Remark 3.3. Further results for the operators $G_{n}^{1}, G_{n}^{2}$, like representation of moments, central moments, images of monomials, quantitative Voronovskaja-type and direct estimates etc. will be subject of another paper. 
Remark 3.4. In [13] Gupta and Lopez-Moreno considered for Phillips operators different Korovkin test system $\left\{1, t, e^{b t}\left(1+t^{r}\right)\right\}$. But in their settings we need to verify the weighted approximation for two test functions with more complicated calculations, than presented in our note.

\section{References}

[1] Acar, T., Aral, A., Gonska, H., On Szász-Mirakyan operators preserving $e^{2 a x}, a>0$, Mediterr. J. Math., 14(6)(2017).

[2] Acar, T., Aral, A., Cárdenas- Morales, D., Garrancho, P., Szász-Mirakyan type operators which fix exponentials, Results Math., 72(2017), no. 3, 1341-1358.

[3] Aral, A., Inoan, D., Raşa, I., On the generalized Szász-Mirakyan operators, Results Math., 65(2014), 441-452.

[4] Aral, A., Inoan, D., Raşa, I., Approximation properties of Szász-Mirakyan operators preserving exponential functions, Positivity (2018) (to appear).

[5] Bodur, M., Yilmaz, O.G., Aral, A., Approximation by Baskakov-Szász-Stancu operators preserving exponential functions, Constr. Math. Anal., 1(2018), no. 1, 1-8.

[6] Boyanov, B.D., Veselinov, V.M., A note on the approximation of functions in an infinite interval by linear positive operators, Bull. Math. Soc. Sci. Math. Roum., 14(62)(1970), 9-13.

[7] Duman, O., Ozarslan, M.A., Szász-Mirakyan type operators providing a better error estimation, Appl. Math. Lett., 20(2007), 1184-1188.

[8] Finta, Z., Gupta, V., Direct and inverse estimates for Phillips type operators, J. Math. Anal. Appl., 303(2005), no. 2, 627-642.

[9] Gadziev, A.D., Theorems of the type of P.P. Korovkin's Theorems, Mat. Zametki, 20(1976), no. 5, 781-786.

[10] Gonska, H., Quantitative Korovkin-type theorems on simultaneous approximation, Math. Z., 186(1984), 419-433.

[11] Gupta, V., A note on modified Phillips operators, Southeast Asian Bulletin of Mathematics, 34(2010), 847-851.

[12] Gupta, V., Tachev, G., On approximation properties of Phillips operators preserving exponential functions, Mediterr. J. Math., 14(2017), no. 4, art. 177.

[13] Gupta, V., Lopez-Moreno, A.J., Phillips operators preserving arbitrary exponential functions $e^{a t}, e^{b t}$, Filomat (2018) (to appear).

[14] Heilmann, M., Tachev, G., Commutativity, direct and strong converse results for Phillips operators, East J. Approx., 17(2011), no. 3, 299-317.

[15] Heilmann, M., Tachev, G., Linear Combinations of Genuine Szász-Mirakjan-Durrmeyer Operators, In: Anastassiou G., Duman O. (eds), Advances in Applied Mathematics and Approximation Theory, Springer Proceedings in Mathematics \& Statistics, Springer, New York, NY, 41(2013), 85-106.

[16] Holhos, A., The rate of approximation of functions in an infinite interval by positive linear operators, Stud. Univ. Babeş-Bolyai Math., 55(2010), no. 2, 133-142.

[17] King, J.P., Positive linear operators which preserve $x^{2}$, Acta Math. Hungar, 99(2003), no. 3, 203-208. 
[18] Paltanea, R., Optimal estimates with moduli of continuity, Results Math., 32(1997), 318-331.

[19] Paltanea, R., Simic, M., General estimates of the weighted approximation on interval $[0, \infty)$ using moduli of continuity, Bull. Transilv. Univ. Braşov Ser. III, 8(57)(2015), no. 2, 93-108.

[20] Phillips, R.S., An inversion formula for Laplace transforms and semi-groups of linear operators, Annals Math., 59(1954), 325-356.

[21] Tachev, G., A global inverse theorem for combinations of Phillips operators, Mediterr. J. Math., 13(2016), no. 5, 2709-2719.

[22] Tachev, G., Pointwise estimate for linear combinations of Phillips operators, J. Classical Analysis, 8(2016), no. 1, 41-51.

[23] Tachev, G., Gupta, V., Aral, A., Voronovskaja's theorem for functions with exponential growth, Georgian Math. J. (to appear).

[24] Tachev, G., Approximation of bounded continuous functions by linear combinations of Phillips operators, Demonstr. Math., 47(2014), no. 3, 662-671.

Gancho Tachev

University of Architecture, Civil Engineering and Geodesy

Department of Mathematics

Hristo Smirnensky, Blvd., 1,

1046 Sofia, Bulgaria

e-mail: gtt_fte@uacg.bg 